\title{
Hypomyces pseudolactifluorum sp. nov. (Hypocreales: Hypocreaceae) on Russula sp. from Yunnan, PR China
}

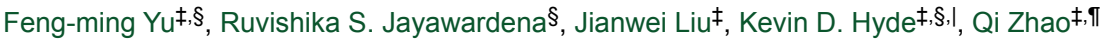 \\ ‡ Key Laboratory of Economic Plants and Biotechnology, Kunming Institute of Botany, Chinese Academy of Sciences, \\ Kunming, China \\ $\S$ Center of Excellence in Fungal Research, Mae Fah Luang University, Chiangrai, Thailand \\ | Institute of Plant Health, Zhongkai University of Agriculture and Engineering, Guangzhou, China \\ II Institute of Applied Fungi, Southwest Forestry University, Kunming, China
}

Corresponding author: Qi Zhao (zhaoqi@mail.kib.ac.cn)

Academic editor: Alfredo Vizzini

Received: 21 Apr 2020 | Accepted: 19 Aug 2020 | Published: 28 Sep 2020

Citation: Yu F, Jayawardena RS, Liu J, Hyde KD, Zhao Q (2020) Hypomyces pseudolactifluorumsp. nov.

(Hypocreales: Hypocreaceae) on Russula sp. from Yunnan, PR China. Biodiversity Data Journal 8: e53490.

https://doi.org/10.3897/BDJ.8.e53490

\begin{abstract}
Background

Hypomyces is a large genus of fungicolous fungi, parasitising the fruiting bodies of Agaricales, Boletales, Helotiales, Pezizales and Polyporales. Hypomyces currently comprises of 147 species widely distributed in Australia, China, France, Germany, Italy, Japan, North America, Sri Lanka, Thailand and UK. Amongst them, 28 species have been recorded in China.
\end{abstract}

\section{New information}

Hypomyces pseudolactifluorum sp. nov., growing on the fruiting bodies of Russula sp. in subsect. Lactarioideae and collected from Yunnan, China, is described with illustrations and molecular phylogenetic data (combined ITS, LSU, TEF1- $\alpha$ and RPB2 sequence dataset). The new species is characterised by semi-immersed to immersed perithecia and 
fusiform, apiculate and verrucose ascospores. We also review the species diversity of the genus Hypomyces in China.

\section{Keywords}

Mycoparasite, species diversity, muti-gene phylogeny

\section{Introduction}

Fungicolous fungi are a large and diverse ecological group, currently containing more than 1500 taxa distributed in many lineages across the fungal kingdom (Põldmaa 2011, Sun et al. 2019a). Hypomyces (Fr.) Tul. \& C. Tul. is an important genus of fungicolous fungi and placed in the family Hypocreaceae (Hypocreales, Sordariomycetes, Ascomycota) (Hyde et al. 2020). Hypomyces was originally introduced as a subgenus of Hypocrea Fr. (Fries 1825) and then Tulasne and Tulasne (1860) revised it to a genus and designated $H$. lactifluorum (Schwein.) Tul. \& C. Tul. from the USA as its type. Hypomyces parasitises the fruiting bodies of Agaricales, Boletales, Helotiales, Pezizales and Polyporales (Rossman et al. 1999, Tamm and Põldmaa 2013, Sun et al. 2019a). Hypomyces is characterised by: superficial or immersed, spherical to ovate, pyriform, papillate and yellow, orange, tawny red or green perithecia in a subiculum; 8-spored, subcylindrical to cylindrical and with a thickened apical asci; and ellipsoid, lanceolate, fusiform to navicular, 0-1-septate or rarely 3-septate, hyaline, spinulose or verrucose and smooth-walled ascospores (Rossman et al. 1999, Zeng and Zhuang 2015). Its allied genera include Cladobotryum Nees, Mycogone Link, Sepedonium Link and Stephanoma Wallr (Wijayawardene et al. 2017) and its asexual morphs are Acremonium-, Dactylaria-, Papulaspora-, Trichothecium- or Verticillium-like (Jaklitsch et al. 2006, Hyde et al. 2020). Hypomyces currently comprises of 147 species in Species Fungorum (http://www.speciesfungorum.org/, accessed in April 2020) and is widely distributed in Australia, China, France, Germany, Italy, Japan, North America, Sri Lanka, Thailand and UK (Zhuang et al. 2012, Rossman et al. 2013, Zeng and Zhuang 2016, Zare and Gams 2016, Lechat et al. 2017, Wei and Kirschner 2017, Sun et al. 2019a, Sun et al. 2019b, Zeng and Zhuang 2019). Amongst them, 28 species have been reported in China (Table 1).

Table 1.

Species diversity of the genus Hypomyces in China (29 species in total).

\begin{tabular}{|l|l|l|l|}
\hline Taxa names & Hosts & Distribution & References \\
\hline $\begin{array}{l}\text { Hypomyces } \\
\text { amaniticola }\end{array}$ & Amanita sp. & China (Yunnan) & Zeng and Zhuang \\
\hline
\end{tabular}




\begin{tabular}{|c|c|c|c|}
\hline Taxa names & Hosts & Distribution & References \\
\hline H. aurantius & $\begin{array}{l}\text { Agaricus bisporus, Polyporales } \\
\text { (Cymatoderma sp., Laetiporus } \\
\text { sulphureus, Panellus sp., Polyporus } \\
\text { picipes), Stereum sp. }\end{array}$ & $\begin{array}{l}\text { China (Anhui, Fujian, } \\
\text { Guangxi, Hainan, Hebei, } \\
\text { Hunan, Jiangsu, Jiangxi, } \\
\text { Shanghai, Sichuan, } \\
\text { Zhejiang), New Zealand, } \\
\text { USA }\end{array}$ & $\begin{array}{l}\text { Chen and Fu } \\
\text { 1989, Põldmaa } \\
\text { 2011, Luo and } \\
\text { Zhuang } 2012\end{array}$ \\
\hline H. aureonitens & Phlebia tremellosa, Polyporus sp. & $\begin{array}{l}\text { China (Fujian, Guangxi), } \\
\text { Europe }\end{array}$ & $\begin{array}{l}\text { Teng 1963, Sun et } \\
\text { al. 2019a }\end{array}$ \\
\hline H. chlorinigenus & Agaricaceae, Boletaceae & $\begin{array}{l}\text { Belgium, China (Taiwan), } \\
\text { Guyana; Indonesia, New } \\
\text { Zealand, USA }\end{array}$ & $\begin{array}{l}\text { Rogerson and } \\
\text { Samuels 1989, } \\
\text { Zeng and Zhuang } \\
2016\end{array}$ \\
\hline H. chrysospermus & $\begin{array}{l}\text { Boletus sp., Hemileccinum impolitum, } \\
\text { Suillus americanus, Russula sp. }\end{array}$ & $\begin{array}{l}\text { China (Fujian, Jiling, } \\
\text { Nanjing), Russia }\end{array}$ & $\begin{array}{l}\text { Ma 2008, Luo and } \\
\text { Zhuang } 2012\end{array}$ \\
\hline H. completiopsis & Boletus sp. & China (Yunnan) & $\begin{array}{l}\text { Zeng and Zhuang } \\
2016\end{array}$ \\
\hline H. fistulina & Fistulina sp. & China (Guangxi) & Sun et al. $2019 b$ \\
\hline H. hubeiensis & Agaricus sp. & China (Hubei) & $\begin{array}{l}\text { Zeng and Zhuang } \\
2019\end{array}$ \\
\hline H. hyalinus & Agaricales (Amanita sp.), Polyporales & $\begin{array}{l}\text { Canada, China (Jiangsu), } \\
\text { Japan, USA }\end{array}$ & $\begin{array}{l}\text { Teng 1934, Teng } \\
1963 \text {, Rogerson } \\
\text { and Samuels } \\
1994\end{array}$ \\
\hline H. lateritius & $\begin{array}{l}\text { Lactarius camphoratus, L. chelidonium, } \\
\text { L. controversus, L. deliciosus, } L \text {. } \\
\text { sanguifluus, L. thejogalus, L. trivialis, } \\
\text { Lactarius sp. }\end{array}$ & $\begin{array}{l}\text { Canada, China (Tibet), } \\
\text { Europe, Japan, Mexico, } \\
\text { New Zealand, USA }\end{array}$ & $\begin{array}{l}\text { Rogerson and } \\
\text { Samuels 1994, } \\
\text { Luo and Zhuang } \\
2012\end{array}$ \\
\hline H. Iuteovirens & $\begin{array}{l}\text { Russula atropurpurea, } R . \text { rosea, } R . \\
\text { sanguinaria, Russula sp. }\end{array}$ & $\begin{array}{l}\text { Canada, China (Inner } \\
\text { Mongolia), Europe, Japan, } \\
\text { Russia, USA }\end{array}$ & $\begin{array}{l}\text { Rogerson and } \\
\text { Samuels } 1994, \\
\text { Ma } 2008\end{array}$ \\
\hline H. macrosporus & Russulaceae & China (Hubei), Mexico, USA & $\begin{array}{l}\text { Rogerson and } \\
\text { Samuels 1994, } \\
\text { Luo and Zhuang } \\
2012\end{array}$ \\
\hline H. microspermus & $\begin{array}{l}\text { Boletaceae, Boletus sp., Imleria badia, } \\
\text { Xanthoconium affine, Xerocomellus } \\
\text { chrysenteron, Xerocomus sp. }\end{array}$ & $\begin{array}{l}\text { Canada, China (Fujian, } \\
\text { Guizhou, Hainan, Hubei, } \\
\text { Jilin, Taiwan, Yunan), } \\
\text { Indonesia, New Zealand, } \\
\text { USA }\end{array}$ & $\begin{array}{l}\text { Rogerson and } \\
\text { Samuels 1989, } \\
\text { Zeng and Zhuang } \\
2016\end{array}$ \\
\hline H. mycophilus & $\begin{array}{l}\text { Auricularia sp., Bulgari sp., Marasmius } \\
\text { sp., Polyporus sp., Trametes versicolor }\end{array}$ & China (Guangdong), USA & $\begin{array}{l}\text { Rogerson and } \\
\text { Samuels } 1993, \\
\text { Zeng et al. } 2017\end{array}$ \\
\hline H. ochraceus & $\begin{array}{l}\text { Decaying leaves, wood and fungi (e.g. } \\
\text { Russula sp.) }\end{array}$ & $\begin{array}{l}\text { China (Guangxi, Yunnan), } \\
\text { Europe, USA }\end{array}$ & $\begin{array}{l}\text { Teng 1963, Sun et } \\
\text { al. 2019a }\end{array}$ \\
\hline H. orthosporus & Polyporales & $\begin{array}{l}\text { China (Tibet), Estonia, } \\
\text { Finland, The Netherlands }\end{array}$ & $\begin{array}{l}\text { Põldmaa 1996, } \\
\text { Zeng and Zhuang } \\
2019\end{array}$ \\
\hline
\end{tabular}




\begin{tabular}{|c|c|c|c|}
\hline Taxa names & Hosts & Distribution & References \\
\hline H. papulasporae & $\begin{array}{l}\text { Geoglossum difforme, G. fallax, G. } \\
\text { glabrum, G. nigritum, G. simile, } \\
\text { Glutinoglossum glutinosum, } \\
\text { Trichoglossum hirsutum, T. walteri }\end{array}$ & China, USA, New Zealand & $\begin{array}{l}\text { Rogerson and } \\
\text { Samuels } 1985, \\
\text { Sun et al. } 2019 a\end{array}$ \\
\hline H. polyporinus & $\begin{array}{l}\text { Auricularia auricula-judae, Polyporales, } \\
\text { Trametes versicolor, T. pubescens, } \\
\text { Polyporus sp. }\end{array}$ & $\begin{array}{l}\text { Canada, China (Guangxi), } \\
\text { USA }\end{array}$ & $\begin{array}{l}\text { Teng } 1963, \\
\text { Rogerson and } \\
\text { Samuels } 1993\end{array}$ \\
\hline $\begin{array}{l}\text { H. } \\
\text { pseudolactifluorum } \\
\text { sp. nov. }\end{array}$ & Russula sp. & China (Yunnan) & This study \\
\hline H. rosellus & $\begin{array}{l}\text { Agaricus bisporus, Armillaria sp., } \\
\text { Hydnellum sp., Hyphoderma sp., } \\
\text { Mycena sp., Polyporus sp., Russula sp., } \\
\text { Trichaptum sp. }\end{array}$ & $\begin{array}{l}\text { China (Gansu), Europe, } \\
\text { Iran, Japan, Korea, USA }\end{array}$ & $\begin{array}{l}\text { Tamm and } \\
\text { Põldmaa } 2013 \text {, } \\
\text { Sun et al. } 2019 b\end{array}$ \\
\hline H. semicircularis & $\begin{array}{l}\text { Ganoderma sichuanense, Microporus } \\
\text { xanthopus }\end{array}$ & Cuba, China & $\begin{array}{l}\text { Wei and } \\
\text { Kirschner 2017, } \\
\text { Sun et al. 2019a }\end{array}$ \\
\hline H. sibirinae & $\begin{array}{l}\text { Aphyllophorales, Boletus sp., } \\
\text { Polyporales }\end{array}$ & $\begin{array}{l}\text { China (Hunan), Indonesia, } \\
\text { USA }\end{array}$ & $\begin{array}{l}\text { Samuels et al. } \\
\text { 1990, Zeng et al. } \\
\text { 2017, Sun et al. } \\
\text { 2019a }\end{array}$ \\
\hline H. sinicus & Schizophyllum sp. & China (Anhui) & $\begin{array}{l}\text { Zhuang et al. } \\
2012\end{array}$ \\
\hline H. stephanomatis & Humaria hemisphaerica, Humaria sp. & $\begin{array}{l}\text { Canada, China (Hubei), } \\
\text { Germany, USA }\end{array}$ & $\begin{array}{l}\text { Rogerson and } \\
\text { Samuels } \\
\text { 1985,Zeng and } \\
\text { Zhuang } 2016\end{array}$ \\
\hline H. subiculosus & $\begin{array}{l}\text { Polyporaceae (Microporus affinis, } \\
\text { Trametes versicolo) }\end{array}$ & $\begin{array}{l}\text { China (Anhui, Beijing, } \\
\text { Guangxi, Zhejiang), Cuba, } \\
\text { Japan }\end{array}$ & $\begin{array}{l}\text { Rogerson and } \\
\text { Samuels 1993, } \\
\text { Luo and Zhuang } \\
2012\end{array}$ \\
\hline H. succineus & Pholiota sp. & China (Taiwan), USA & $\begin{array}{l}\text { Rogerson and } \\
\text { Samuels 1994, } \\
\text { Zeng and Zhuang } \\
2016\end{array}$ \\
\hline H. tegillum & Aphyllophorales, Polyporales & $\begin{array}{l}\text { Brazil, China (Guangxi, } \\
\text { Yunnan), Panama, USA }\end{array}$ & $\begin{array}{l}\text { Rogerson and } \\
\text { Samuels 1993, } \\
\text { Luo and Zhuang } \\
2012\end{array}$ \\
\hline H. triseptatus & $\begin{array}{l}\text { Bark or associated with an ascomycete; } \\
\text { Pyrenomycete }\end{array}$ & $\begin{array}{l}\text { China (Hunan, Guangdong), } \\
\text { Gabon }\end{array}$ & $\begin{array}{l}\text { Rossman and } \\
\text { Rogersson } 1981 \text {, } \\
\text { Zeng et al. } 2017\end{array}$ \\
\hline H. yunnanensis & Boletus sp. & China (Yunnan) & $\begin{array}{l}\text { Zeng and Zhuang } \\
2016\end{array}$ \\
\hline
\end{tabular}

Fungicolous fungi play important roles in the processes of the growths and degradations of their hosts. With the rapid development of mushroom industries, the fungicolous fungi on mushrooms have received more and more attention (Hyde et al. 2019). In this paper, we 
introduce a new member of fungicolous fungi, Hypomyces pseudolactifluorum sp. nov., on the fruiting bodies of Russula sp., collected from Yunnan Province, China. At the same time, we review the species diversity of the genus Hypomyces in China.

\section{Materials and methods}

\section{Collections and Morphology}

Hypomyces specimens, including their host mushrooms, were collected in an evergreen broad-leaved forest in Baihualing, Baoshan, Yunnan Province, China. The specimens, as well as collected host mushrooms, were placed on a piece of aluminium foil at first, then rolled the paper into a cylinder, twisted at the ends for sealing and lastly taken back to the laboratory for study (McKnight and McKnight 1997). Colour codes were recorded following those of Kornerup and Wanscher (1978). A Nikon Coolpix P510 camera was used to take photos in the wild. Dried specimens were observed and photographed using an Olympus SZ61 stereomicroscope and a Nikon ECLIPSE Ni compound microscope fitted with a Canon EOS 600D digital camera. Measurements were made using the Tarosoft $\circledast$ Image Frame Work programme v.0.9.7. The colour change of the perithecial wall was tested using $5 \%$ potassium hydroxide $(\mathrm{KOH})$. Type specimens are deposited at the Herbarium of Mae Fah Luang University, Thailand (MFLU) and the Herbarium of Cryptogams Kunming Institute of Botany, Chinese Academy of Sciences, PR China (HKAS).

\section{DNA extraction, PCR amplification and sequencing}

The genomic DNA was extracted from the dried materials using the CTAB method (Doyle 1987). Tissues from the ascocarps of parasitic fungi and fruiting bodies of the host mushrooms were used to extract DNA, respectively. Primer pairs ITS1F/ITS4 (White et al. 1990), LROR/LR5 (Rehner and Samuels 1994, Vilgalys and Hester 1990), TEF1- $\alpha$ 983f/ TEF1- $\alpha$ 2218r (Carbone and Kohn 1999, Rehner and Buckley 2005) and RPB2-5f/ RPB2-7cR (Liu et al. 1999) were used for amplification of the ITS, LSU, TEF1- $\alpha$ and RPB2 gene regions.

PCR was performed in a $25 \mu$ reaction volume: $12.5 \mu \mathrm{l}$ Taq PCR Master Mix (Abmgood, Richmond, BC, Canada), $1 \mu$ forward primer, $1 \mu$ reverse primer, $1 \mu$ l DNA template and $9.5 \mu \mathrm{lddH} \mathrm{H}_{2} \mathrm{O}$. For ITS and LSU, PCR reaction conditions are: $8 \mathrm{~min}$ at $94^{\circ} \mathrm{C}$, followed by $30 \mathrm{~s}$ at $94^{\circ} \mathrm{C}, 30 \mathrm{~s}$ at $52^{\circ} \mathrm{C}$ and $1 \mathrm{~min}$ at $72^{\circ} \mathrm{C}$ for 35 cycles and a final extension of $10 \mathrm{~min}$ at $72^{\circ} \mathrm{C}$. PCR reaction conditions of TEF1- $\alpha$ and RPB2 are: $8 \mathrm{~min}$ at $94^{\circ} \mathrm{C}$, followed by 1 min at $95^{\circ} \mathrm{C}, 45 \mathrm{~s}$ at $59^{\circ} \mathrm{C}$ for RPB2 $/ 55^{\circ} \mathrm{C}$ for $T E F 1-\alpha$ and $1 \mathrm{~min}$ at $72^{\circ} \mathrm{C}$ for 35 cycles and a final extension of $10 \mathrm{~min}$ at $72^{\circ} \mathrm{C}$. The PCR products were detected using agarose gel electrophoresis and, in the gel documentation system, clear bands were observed. Sequencing was performed by Sangon Biotech (Shanghai) Co. Ltd., PR China; partial impure products were purified using the Cycle-pure-kit (Omega, America) and then cloned into pClone007 Simple vector (TSV-007S from Beijing TsingKe Biotech). Twenty clones of PCR products of each gene were sequenced using the universal primer pairs M13-47/ M13-48. 


\section{Sequence alignment and phylogenetic analyses}

\section{The parasitic fungus: Hypomyces pseudolactifluorum sp. nov.}

Molecular phylogenetic trees were constructed using our sequencing results of $H$. pseudolactifluorum sp. nov. and the voucher sequences of their allies obtained from NCBI GenBank (Table 2). Two species of Trichoderma, T. hamatum (DAOM 167057) and T. viride (CBS 119325) were used as outgroup taxa. All sequences were assembled and aligned using MAFFT v6.8 (Katoh et al. 2005) and manually edited via BioEdit version 7.0.9 (Hall 1999). Four sequence matrices of ITS, LSU, TEF1- $\alpha$ and RPB2 genes, respectively, were compiled. The optimal substitution model for each gene dataset was determined using jModelTest2 under the Akaike information criterion (AIC) (Darriba et al. 2012). The results indicated that the $G T R+I+G$ model $(-\operatorname{InL}=8658.2624)$ is optimal for the ITS dataset, as well as the TIM1+I+G model $(-\operatorname{InL}=4392.5417)$ for $L S U$, the $\operatorname{TrN}+I+G$ model $(-\operatorname{InL}=5751.4959)$ for TEF1- $\alpha$ and the model SYM+I+G $(-\operatorname{InL}=6419.6669)$ for RPB2, respectively. Using the aligned sequence matrices, a combined gene sequence dataset (ITS, LSU, TEF1- $\alpha$ and RPB2, orderly) was assembled and aligned and was finally deposited in TreeBASE database (http://purl.org/phylo/treebase/phylows/study/ TB2:S26593? x-access-code=152eadfc2292343af7627cfad5c2946c\&format=html).

Table 2.

Voucher information and GenBank accession numbers for samples appearing in the Hypomyces phylogenetic tree. Our sequencing results are displayed in bold. (Label T indicate the sequences from ex-type strains.)

\begin{tabular}{|c|c|c|c|c|c|c|}
\hline \multirow[t]{2}{*}{ Taxa names } & \multirow{2}{*}{$\begin{array}{l}\text { Specimen/ } \\
\text { Strain } \\
\text { number }\end{array}$} & \multicolumn{4}{|c|}{ GenBank accession numbers } & \multirow[t]{2}{*}{ References } \\
\hline & & ITS & LSU & TEF1- $\alpha$ & RPB2 & \\
\hline $\begin{array}{l}\text { Cladobotryum } \\
\text { amazonense }\end{array}$ & CBS 470.80 & $\underline{\mathrm{MH} 861285}$ & MH873051 & I & I & Vu et al. 2019 \\
\hline C. apiculatum & CBS $174.56^{\top}$ & NR 159770 & MH869109 & I & l & Vu et al. 2019 \\
\hline C. asterophorum & CBS $676.77^{\top}$ & FN859395 & MH872869 & FN868712 & FN868649 & Põldmaa 2011 \\
\hline C. croceum & CBS 231.95 & MH862511 & MH874154 & I & I & Vu et al. 2019 \\
\hline \multirow[t]{2}{*}{ C. cubitense } & CBS 416.85 & FN859396 & I & FN868713 & FN868650 & Põldmaa 2011 \\
\hline & G.A. m643.w & FN859397 & I & FN868714 & $\underline{F N 868651}$ & Põldmaa 2011 \\
\hline C. indoafrum & TFC 201295 & FN859403 & $\underline{F N 859403}$ & FN868721 & FN868657 & Põldmaa 2011 \\
\hline C. multiseptatum & CBS $472.71^{\top}$ & FN859405 & MH871991 & FN868723 & FN868659 & Põldmaa 2011 \\
\hline C. obconicum & CBS 528.81 & $\underline{\mathrm{MH} 861373}$ & $\underline{\mathrm{MH} 873126}$ & I & l & Vu et al. 2019 \\
\hline C. paravirescens & TFC $97-23^{\top}$ & FN859406 & FN859406 & FN868724 & FN868660 & Põldmaa 2011 \\
\hline C. penicillatum & CBS $407.80^{\top}$ & FN859407 & MH873046 & FN868725 & FN868661 & Põldmaa 2011 \\
\hline C. protrusum & CBS 118999 & FN859408 & FN859408 & FN868726 & FN868662 & Põldmaa 2011 \\
\hline C. purpureum & CBS $154.78^{\top}$ & FN859415 & I & FN868733 & FN868669 & Põldmaa 2011 \\
\hline C. rubrobrunnescens & CBS $176.92^{\top}$ & FN859416 & MH874016 & FN868734 & FN868670 & Põldmaa 2011 \\
\hline
\end{tabular}




\begin{tabular}{|c|c|c|c|c|c|c|}
\hline \multirow[t]{2}{*}{ Taxa names } & \multirow{2}{*}{$\begin{array}{l}\text { Specimen/ } \\
\text { Strain } \\
\text { number }\end{array}$} & \multicolumn{4}{|c|}{ GenBank accession numbers } & \multirow[t]{2}{*}{ References } \\
\hline & & ITS & LSU & TEF1- $\alpha$ & RPB2 & \\
\hline Cladobotryum sp. & FSU 5046 & FN859421 & I & FN868739 & FN868675 & Põldmaa 2011 \\
\hline C. stereicola & CBS $457.71^{\top}$ & $\underline{\mathrm{MH} 860217}$ & MH871984 & I & 1 & Vu et al. 2019 \\
\hline C. tchimbelense & TFC $201146^{\top}$ & FN859419 & FN859419 & FN868737 & FN868673 & Põldmaa 2011 \\
\hline C. tenue & CBS $152.92^{\top}$ & FN859420 & FN859420 & FN868738 & FN868674 & Põldmaa 2011 \\
\hline \multirow[t]{2}{*}{ Hypomyces aconidialis } & TFC $201334^{\top}$ & FN859457 & FN859457 & FN868775 & FN868711 & Põldmaa 2011 \\
\hline & TFC 201215 & $\underline{\text { FN859456 }}$ & $\underline{\text { FN859456 }}$ & FN868774 & FN868710 & Põldmaa 2011 \\
\hline H. albidus & CBS 460.71 & $\underline{\mathrm{MH} 860220}$ & $\underline{\text { MH871987 }}$ & I & 1 & Vu et al. 2019 \\
\hline H. armeniacus & TFC $02-86 / 2^{\top}$ & FN859424 & FN859424 & FN868742 & FN868678 & Põldmaa 2011 \\
\hline H. aurantius & TFC 95-171 & FN859425 & $\underline{\text { FN859425 }}$ & FN868743 & FN868679 & Põldmaa 2011 \\
\hline \multirow[t]{2}{*}{ H. australasiaticus } & TFC 03-8 ${ }^{\top}$ & $\underline{\text { FN859428 }}$ & $\underline{\text { FN859428 }}$ & FN868746 & FN868681 & Põldmaa 2011 \\
\hline & TFC 99-95 & FN859427 & I & FN868745 & FN868680 & Põldmaa 2011 \\
\hline H. australis & TFC 2007-18 & $\underline{\mathrm{AM} 779860}$ & $\underline{\mathrm{AM} 779860}$ & $\underline{\text { FN868747 }}$ & I & Põldmaa 2011 \\
\hline H. boletiphagus & $\begin{array}{l}\text { MFLU } \\
17-1391\end{array}$ & $\underline{\mathrm{MH} 459152}$ & $\underline{\mathrm{MH} 459168}$ & I & $\underline{\mathrm{MH} 464785}$ & Sun et al. $2019 \mathrm{~b}$ \\
\hline H. boletus & $\begin{array}{l}\text { MFLU } \\
17-1392\end{array}$ & $\underline{\mathrm{MH} 459153}$ & $\underline{\mathrm{MH} 459170}$ & I & $\underline{\mathrm{MH} 464787}$ & Sun et al. $2019 b$ \\
\hline \multirow[t]{2}{*}{ H. chlorinigenus } & KSH511 & $\underline{\text { KT946843 }}$ & I & $\underline{\text { KU041505 }}$ & KU041493 & Otto et al. 2016 \\
\hline & $\mathrm{KSH} 512^{\top}$ & KT946844 & I & $\underline{\text { KU041506 }}$ & $\underline{\text { KU041494 }}$ & Otto et al. 2016 \\
\hline \multirow[t]{2}{*}{ H. completus } & $\begin{array}{l}\text { KSH411 } \\
\text { (S172) }\end{array}$ & $\underline{\text { KT946842 }}$ & I & $\underline{\text { KU041504 }}$ & KU041492 & Otto et al. 2016 \\
\hline & $\begin{array}{l}\mathrm{KSH} 410 \\
(\mathrm{~S} 171)^{\top}\end{array}$ & $\underline{\text { KT946841 }}$ & I & $\underline{\text { KU041503 }}$ & $\underline{\text { KU041491 }}$ & Otto et al. 2016 \\
\hline H. corticiicola & CBS $137.71^{\top}$ & MH860037 & MH871817 & I & I & Vu et al. 2019 \\
\hline H. dactylarioides & CBS $141.78^{\top}$ & FN859429 & MH872879 & FN868748 & FN868683 & Põldmaa 2011 \\
\hline H. fistulina & $\begin{array}{l}\text { HMAS } \\
279800^{\top}\end{array}$ & $\underline{\mathrm{MH} 459154}$ & $\underline{\mathrm{MH} 459171}$ & $\underline{\mathrm{MH} 464781}$ & 1 & Sun et al. 2019b \\
\hline H. gabonensis & TFC $201156^{\top}$ & FN859430 & FN859430 & FN868749 & FN868684 & Põldmaa 2011 \\
\hline H. heterosporus & CBS $719.88^{\top}$ & $\underline{\text { FN859398 }}$ & $\underline{\mathrm{MH} 873844}$ & FN868716 & $\underline{\text { FN868653 }}$ & Põldmaa 2011 \\
\hline H. khaoyaiensis & $\begin{array}{l}\text { G.J.S. 01-304 } \\
T\end{array}$ & FN859431 & AJ583483 & FN868750 & FN868685 & Põldmaa 2011 \\
\hline H. lactifluorum & $\begin{array}{l}\text { TAAM } 170476 \\
T\end{array}$ & FN859432 & EU710768 & FN868751 & EU710773 & Põldmaa 2011 \\
\hline H. laeticolor & JCM $10758^{\top}$ & $\underline{\text { LC228655 }}$ & $\underline{\text { LC228712 }}$ & I & 1 & Sun et al. $2019 b$ \\
\hline H. luteovirens & CBS 128483 & $\underline{\mathrm{MH} 864958}$ & $\underline{\mathrm{MH} 876402}$ & I & I & Vu et al. 2019 \\
\hline H. mycophilus & CBS 175.56 & $\underline{\mathrm{MH} 857567}$ & $\underline{\text { MH869110 }}$ & I & 1 & Vu et al. 2019 \\
\hline \multirow[t]{2}{*}{ H. odoratus } & G.A. m329 & FN859434 & $\underline{\text { FN859434 }}$ & FN868753 & $\underline{F N 868688}$ & Põldmaa 2011 \\
\hline & TFC 200887 & FN859439 & l & FN868757 & FN868693 & Põldmaa 2011 \\
\hline
\end{tabular}




\begin{tabular}{|c|c|c|c|c|c|c|}
\hline \multirow[t]{2}{*}{ Taxa names } & \multirow{2}{*}{$\begin{array}{l}\text { Specimen/ } \\
\text { Strain } \\
\text { number }\end{array}$} & \multicolumn{4}{|c|}{ GenBank accession numbers } & \multirow[t]{2}{*}{ References } \\
\hline & & ITS & LSU & TEF1- $\alpha$ & RPB2 & \\
\hline H. orthosporus & 10736 & MK478468 & $\underline{\mathrm{MN} 044763}$ & $\underline{\text { MK484609 }}$ & I & $\begin{array}{l}\text { Zeng and Zhuang } \\
2019\end{array}$ \\
\hline H. penicillatus & $\begin{array}{l}\text { NBRC } \\
100524\end{array}$ & $\underline{\mathrm{LC} 146740}$ & $\underline{\text { LC146740 }}$ & 1 & I & Sun et al. $2019 b$ \\
\hline \multirow[t]{2}{*}{$\begin{array}{l}\text { H. pseudolactifluorum } \\
\text { sp.nov. }\end{array}$} & $\begin{array}{l}\text { MFLU } \\
20-0265^{\top}\end{array}$ & MT260402 & MT260399 & MT259361 & MT259359 & This study \\
\hline & $\begin{array}{l}\text { MFLU } \\
20-0266\end{array}$ & MT260403 & MT260400 & MT259362 & MT259360 & This study \\
\hline H. polyporinus & ATCC 76479 & AF543771 & AF543793 & AF543784 & 1 & Currie et al. 2003 \\
\hline H. pseudocorticiicola & $\mathrm{JCM} 12654^{\top}$ & $\underline{\mathrm{LC} 228663}$ & $\underline{\text { LC228721 }}$ & 1 & l & Sun et al. $2019 b$ \\
\hline H. rosellus & TFC 201071 & FN859443 & $\underline{\text { FN859443 }}$ & FN868762 & FN868697 & Põldmaa 2011 \\
\hline \multirow[t]{2}{*}{ H. samuelsii } & CBS 536.88 & FN859444 & l & $\underline{\text { FN868763 }}$ & FN868698 & Põldmaa 2011 \\
\hline & TFC 2007-23 & FN859451 & FN859451 & $\underline{\text { FN868769 }}$ & FN868705 & Põldmaa 2011 \\
\hline H. semicircularis & CBS $705.88^{\top}$ & FN859417 & $\underline{\mathrm{MH} 873843}$ & FN868735 & FN868671 & Põldmaa 2011 \\
\hline \multirow[t]{2}{*}{ H. semitranslucens } & CBS 458.71 & MH860218 & MH871985 & 1 & I & Vu et al. 2019 \\
\hline & CBS 821.70 & MH859960 & MH871759 & I & I & Vu et al. 2019 \\
\hline H. sibirinae & CBS 744.88 & MH862151 & AJ459304 & I & I & Vu et al. 2019 \\
\hline H. sinicus & $\begin{array}{l}\text { HMAS } \\
251317^{\top}\end{array}$ & NR 156252 & $\underline{\text { MN044986 }}$ & $\underline{\text { MK484610 }}$ & 1 & Zhuang et al. 2012 \\
\hline H. stephanomatis & G.J.S. $88-50$ & I & AF160243 & AF534632 & AF545566 & $\begin{array}{l}\text { Põldmaa et al. } \\
2000\end{array}$ \\
\hline H. subiculosus & TFC 97.166 & FN859452 & I & FN868770 & FN868706 & Põldmaa 2011 \\
\hline \multirow[t]{2}{*}{ H. tubariicola } & CBS $115.79^{\top}$ & $\underline{\text { KU382164 }}$ & $\underline{\mathrm{MH} 872953}$ & I & I & Vu et al. 2019 \\
\hline & CBS 225.84 & KU382162 & KU382220 & 1 & I & $\begin{array}{l}\text { Zare and Gams } \\
2016\end{array}$ \\
\hline \multirow[t]{2}{*}{ H. virescens } & G.A. i1906 ${ }^{\top}$ & FN859454 & I & FN868772 & FN868708 & Põldmaa 2011 \\
\hline & G.A. i1899 & FN859453 & I & FN868771 & FN868707 & Põldmaa 2011 \\
\hline $\begin{array}{l}\text { Sepedonium } \\
\text { ampullosporum }\end{array}$ & CBS $392.52^{\top}$ & MH857094 & $\underline{\mathrm{MH} 868629}$ & 1 & I & Vu et al. 2019 \\
\hline \multirow[t]{2}{*}{ S. chalcipori } & CBS 278.92 & MH862358 & $\underline{\mathrm{MH} 874023}$ & 1 & I & Vu et al. 2019 \\
\hline & CBS $148.92^{\top}$ & MH862347 & $\underline{\mathrm{MH} 874014}$ & 1 & I & Vu et al. 2019 \\
\hline S. tulasneanum & CBS 940.69 & MH859489 & $\underline{\mathrm{MH} 871270}$ & 1 & I & Vu et al. 2019 \\
\hline Trichoderma hamatum & $\begin{array}{l}\text { DAOM } \\
167057^{\top}\end{array}$ & EU280124 & $\underline{\mathrm{HM} 466686}$ & AF534620 & AF545548 & $\begin{array}{l}\text { Hoyos-Carvajal et } \\
\text { al. } 2009\end{array}$ \\
\hline T. viride & CBS $119325^{\top}$ & DQ677655 & I & DQ672615 & EU711362 & $\begin{array}{l}\text { Jaklitsch et al. } \\
2006\end{array}$ \\
\hline
\end{tabular}

Maximum Likelihood (ML) analysis was performed using IQ-Tree (Nguyen et al. 2014, Chernomor et al. 2016) with the computing models listed above and a bootstrap test of 
1000 replicates. Bayesian Inference $(\mathrm{BI})$ analysis was carried out using MrBayes v3.2.6 (Ronquist et al. 2012). The TIM1 and TrN substitution models were replaced by the GTR model (Huelsenbeck and Rannala 2004). Four simultaneous Markov Chain Monte Carlo (MCMC) chains were run for random trees of 10,000,000 generations and were sampled by every 100 generations. The computing was stopped when the standard deviation of the split frequencies fell below 0.01 and ESS values $>200$. Subsequently, phylogenetic trees were summarised and posterior probabilities (PP) were performed using MCMC by discarding the first $25 \%$ generations as "burn-in" (Huelsenbeck and Ronquist 2001). Gaps were treated as missing data. Phylogenetic trees were viewed in FigTree v.1.4.2 (http://tree.bio.ed.ac.uk/software/figtree).

\section{The host mushroom: Russula sp.}

Voucher sequences (ITS gene) for phylogenetic analyses of the host mushroom and its allies were obtained from our sequencing results and GenBank databases (Li et al. 2020) (Table 3). Five species of Russula subg. Compactae, $R$. acrifolia, $R$. adusta, $R$. eccentrica, $R$. nigricans and $R$. subnigricans were selected as the outgroup taxa. Sequence alignment and phylogenetic analyses followed those of the parasitic fungus above. ML analysis was performed using IQ-Tree with TVM+I+G model (-InL = 5298.7964) (Nguyen et al. 2014, Chernomor et al. 2016). The ITS sequence matrix of the host mushroom and its allies were deposited in the TreeBASE database (http://purl.org/phylo/treebase/phylows/study/ TB2:S26693?x-access-code=2e445b17aebe1f93266051a8920ae62f\&format=html).

Table 3.

Voucher information and GenBank accession numbers for samples appearing in the Russula phylogenetic tree. Our sequencing results are displayed in bold.

\begin{tabular}{|c|c|c|c|}
\hline Taxa names & Specimen/Strain number & GenBank accession & References \\
\hline Russula acrifolia & TUB UE12.09.2003-3 & $\underline{\mathrm{DQ} 421998}$ & Eberhardt 2002 \\
\hline R. adusta & PC 547RUS27 & AY061652 & Miller and Buyck 2002 \\
\hline$R$. aff. chloroides & FH 12273 & KT934015 & Looney et al. 2016 \\
\hline R. brevipes & SMI329 & FJ845429 & Kranabetter et al. 2009 \\
\hline R. brevipes & JS160927-01 & MG407682 & GenBank \\
\hline R. brevipes & TENN 070667 & $\underline{\mathrm{KY} 848511}$ & Looney et al. 2018 \\
\hline R. brevipes var. acrior & JMP 0058 & EU819422 & Palmer et al. 2008 \\
\hline R. byssina & HGAS-MF 009907 & MN648951 & Li et al. 2020 \\
\hline R. byssina & HGAS-MF009921 & MN648949 & Li et al. 2020 \\
\hline R. byssina & HGAS-MF 009913 & MN648950 & Li et al. 2020 \\
\hline R. cascadensis & UBC F30189 & $\underline{K X 812838}$ & Bazzicalupo 2018 \\
\hline R. cascadensis & UBC F19691 & $\underline{\mathrm{HM} 240541}$ & Buyck et al. 2017 \\
\hline R. cf. angustispora & PC BB2004-252 & EU598152 & GenBank \\
\hline R. cf. brevipes & F 28785 & MH718203 & GenBank \\
\hline
\end{tabular}




\begin{tabular}{|c|c|c|c|}
\hline Taxa names & Specimen/Strain number & GenBank accession & References \\
\hline R. cf. brevipes & F CDW47 & GQ166868 & GenBank \\
\hline R. cf. brevipes & GO 2009-276 & KC152212 & GenBank \\
\hline R. cf. delica & UBC F30260 & KX812852 & Bazzicalupo 2018 \\
\hline R. chloroides & PC 205RUS24 & AY061663 & Miller and Buyck 2002 \\
\hline R. chloroides & UBC F20353 & KC581331 & GenBank \\
\hline R. chloroides & RUS-12091401 & KF432954 & Wisitrassameewong et al. 2014 \\
\hline R. cremicolor & HGAS-MF 009901 & MN648955 & Li et al. 2020 \\
\hline R. cremicolor & HGAS-MF 009908 & MN648952 & Li et al. 2020 \\
\hline R. cremicolor & HGAS-MF 009912 & MN648953 & Li et al. 2020 \\
\hline R. cremicolor & HGAS-MF 009919 & MN648954 & Li et al. 2020 \\
\hline R. delica & hue22 (TUB) & $\underline{\mathrm{AF} 418605}$ & Eberhardt 2002 \\
\hline R. delica & FH 12-272 & KF432955 & Wisitrassameewong et al. 2014 \\
\hline R. delica & HA 2015-004 & $\underline{\mathrm{KX} 263000}$ & Aghajani et al. 2017 \\
\hline R. delica & PC 496RUS26 & AY061671 & Miller and Buyck 2002 \\
\hline R. delica & TUB hue22 & $\underline{\text { AF418605 }}$ & Eberhardt 2002 \\
\hline R. delica & UBC F30263 & KX812842 & Bazzicalupo 2018 \\
\hline R. delica & RMUKK 37 & $\underline{\mathrm{KX} 267630}$ & GenBank \\
\hline R. delica & KA $12-1327$ & $\underline{\mathrm{KR} 673555}$ & Kim et al. 2015 \\
\hline R. delica & HMJAU 32182 & $\underline{\text { KX094989 }}$ & Liu et al. 2017 \\
\hline R. eccentrica & HCCN 23685 & KC699778 & Park et al. 2014 \\
\hline R. japonica & MHHNU 31049 & MK167414 & Chen and Zhang 2019 \\
\hline R. japonica & HGAS-MF 009923 & MN648957 & Li et al. 2020 \\
\hline R. japonica & HGAS-MF 009915 & MN648956 & Li et al. 2020 \\
\hline R. leucocarpa & HGAS-MF 009910 & MN648948 & Li et al. 2020 \\
\hline R. leucocarpa & HGAS-MF 009916 & $\underline{\text { MN648947 }}$ & Li et al. 2020 \\
\hline R. littoralis & PC 1222IS87 & AY061702 & Miller and Buyck 2002 \\
\hline R. marangania & MEL 2293694 & EU019930 & Lebel and Tonkin 2007 \\
\hline R. nigricans & TUB fo46761 & $\underline{\text { AF418607 }}$ & Eberhardt 2002 \\
\hline R. pallidospora & PC 2-1221IS 85 & AY061701 & Miller and Buyck 2002 \\
\hline R. pumicoidea & MEL T-14771 & EU019931 & Lebel and Tonkin 2007 \\
\hline R. sinuata & MEL H4755 & EU019943 & Lebel and Tonkin 2007 \\
\hline R. subnigricans & MHHNU ZP6932 & EF534351 & Yin et al. 2008 \\
\hline R. vesicatoria & PC 0124666 & $\underline{\text { KY800359 }}$ & Buyck et al. 2017 \\
\hline Russula sp. & MFLU 20-0265 (host) & MT755627 & In this study \\
\hline
\end{tabular}




\section{Taxon treatment}

\section{Hypomyces pseudolactifluorum F. M. Yu, Q. Zhao \& K. D. Hyde, sp. nov.}

\section{Materials}

Holotype:

a. $\quad$ scientificName: Hypomyces pseudolactifluorum; country: China; stateProvince: Yunnan; locality: Baoshan, Longyang, Baihualing; verbatimElevation: 2094m; locationRemarks: label transliteration: "Yunnan, Baoshan, Longyang, Baihualing, on Russula sp., 20 July 2018, Jian-Wei Liu; [云南保山百花岭 $2094 \mathrm{~m}, 2018.07 .20$, 刘建伟]; verbatimCoordinates: $25^{\circ} 17.931$ 'N, $98^{\circ} 47.0718^{\prime} \mathrm{E}$; decimalLatitude: 25.2989; decimalLongitude: 98.7845 ; georeferenceProtocol: label; lifeStage: Telemorph; catalogNumber: MFLU 20-0265; recordedBy: Jian-Wei Liu; identifiedBy: Feng-Ming Yu; dateldentified: 2019

\section{Paratype:}

a. scientificName: Hypomyces pseudolactifluorum; country: China; stateProvince: Yunnan; locality: Baoshan, Longyang, Baihualing; verbatimElevation: 2094m; locationRemarks: label transliteration: "Yunnan, Baoshan, Longyang, Baihualing, on Russula sp., 20 July 2018, Jian-Wei Liu; [云南保山百花岭 2094 m, 2018.07.20, 刘建伟]; verbatimCoordinates: $25^{\circ} 17.931$ 'N, $98^{\circ} 47.0718^{\prime} \mathrm{E}$; decimalLatitude: 25.2989; decimalLongitude: 98.7845 ; georeferenceProtocol: label; lifeStage: Telemorph; catalogNumber: MFLU 20-0266; recordedBy: Jian-Wei Liu; identifiedBy: Feng-Ming Yu; dateldentified: 2019

Isotype:

a. scientificName: Hypomyces pseudolactifluorum; country: China; stateProvince: Yunnan; locality: Baoshan, Longyang, Baihualing; verbatimElevation: 2094m; locationRemarks: label transliteration: "Yunnan, Baoshan, Longyang, Baihualing, on Russula sp., 20 July 2018, Jian-Wei Liu; [云南保山百花岭 2094 m, 2018.07.20, 刘建伟]; verbatimCoordinates: $25^{\circ} 17.931$ 'N, $98^{\circ} 47.0718^{\prime} \mathrm{E}$; decimalLatitude: 25.2989; decimalLongitude: 98.7845 ; georeferenceProtocol: label; lifeStage: Telemorph; catalogNumber: HKAS 107300; recordedBy: Jian-Wei Liu; identifiedBy: Feng-Ming Yu; dateldentified: 2019

\section{Description}

\section{Index Fungorum number: IF557817}

Sexual morph. Subiculum light yellow (4A4-5) when fresh and pale orange, light orange to brownish-orange (5A3-4, 5C4, 6C6) after being dried, usually covering the pileus, stipe and deformed gills of the host mushroom. Perithecia aggregated, semiimmersed to immersed in subiculum, except for their erumpent papilla, yellowish-brown to dark brown (5E6, 6E6, 6F6-8), pyriform to subglobose, 262-484 × 136-284 $\mu \mathrm{m}$; perithecial wall 12-25 $\mu \mathrm{m}$ thick, single-layer, cells 9-22 $\times 4-8 \mu \mathrm{m}$. Papilla prominent, 129-177 $\mu \mathrm{m}$ high, at base 135-284 $\mu \mathrm{m}$ wide. Asci 8-spored, cylindrical, 147-222 $\times 4$ $9 \mu \mathrm{m}$; apex thickened, 4.9-6.0 wide and 2.5-3.0 $\mu \mathrm{m}$ high. Ascospores uniseriate and with ends overlapping, fusiform, 30-38 $\times 6-8 \mu \mathrm{m}$, single-septate, septum median and with dense verrucae and prominently apiculate, apiculi 4.5-8.0 $\mu \mathrm{m}$ long, straight or curved. Asexual morph: unknown. (Fig. 1) 


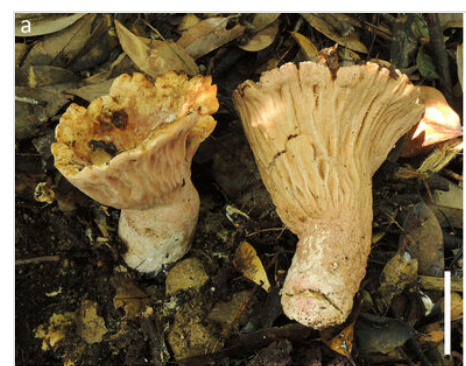

e
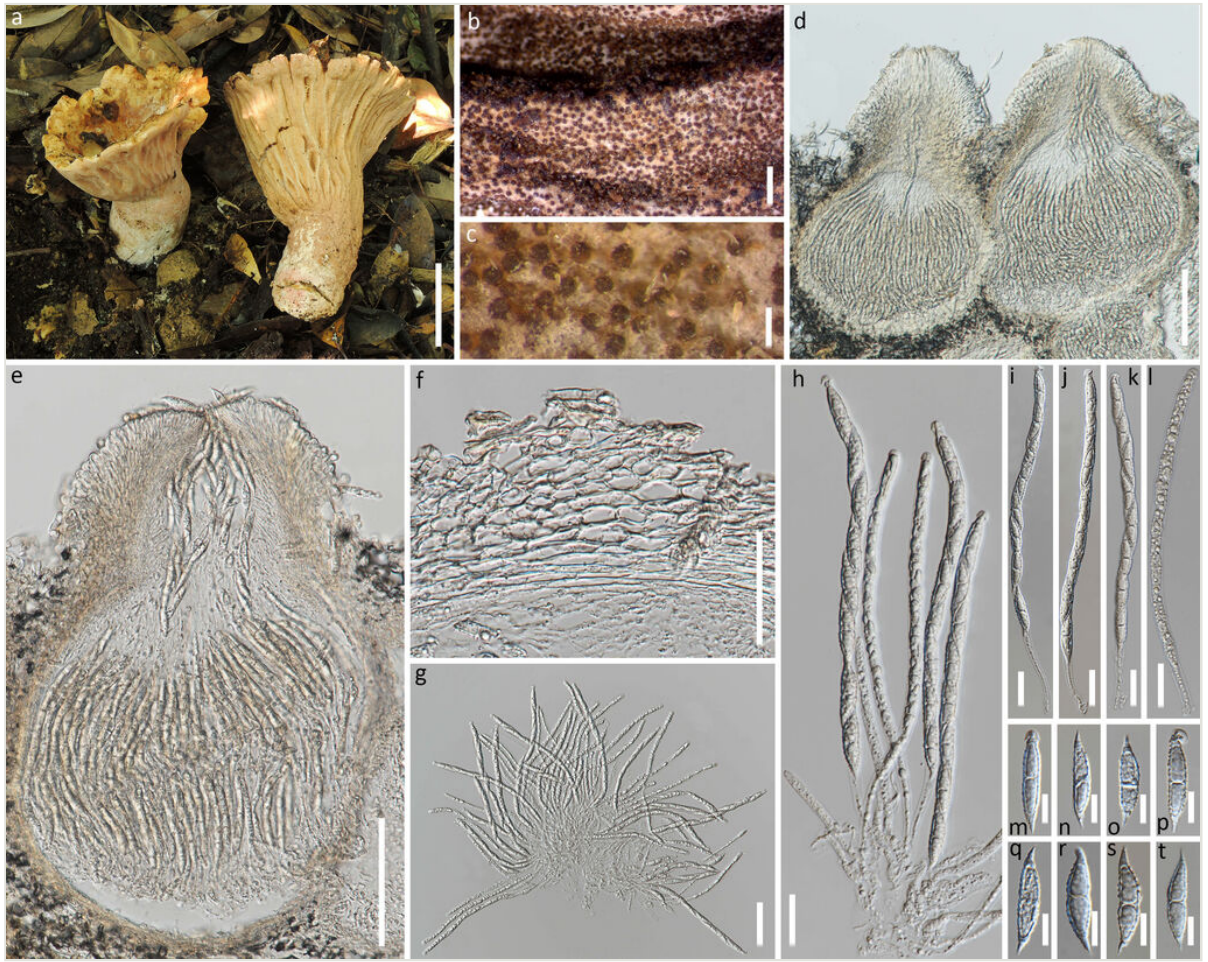

Figure 1. doi

Hypomyces pseudolactifluorum sp. nov.. a: The host mushroom (Russula sp.); b-e: Perithecia embedded in subiculum effused over the substratum; $d-e$ : Median sections of an ascoma; $f$ : Section of peridium; g-l: Asci with ascospores; $m$-t: Ascospores. Scale bars: $a=5 \mathrm{~cm} ; b=1$ $\mathrm{mm} ; \mathrm{c}=200 \mu \mathrm{m} ; \mathrm{d}, \mathrm{e}=100 \mu \mathrm{m} ; \mathrm{f}, \mathrm{g}=50 \mu \mathrm{m} ; \mathrm{h}-\mathrm{I}=20 \mu \mathrm{m} ; \mathrm{m}-\mathrm{t}=10 \mu \mathrm{m}$.

\section{Diagnosis}

The new species is similar to Hypomyces lactifluorum on Russula and Lactarius spp. from North America (Rogerson and Samuels 1994), but has smaller perithecia and shorter asci. The main differences of the two species are compared in Table 4.

Table 4.

Main differences between Hypomyces lactifluorum and $H$. pseudolactifluorum sp. nov..

\section{H. lactifluorum}

\section{H. pseudolactifluorum}

(Rogerson and Samuels 1994)

Subiculum Pale yellowish-orange to bright orange (young), in age becoming deep red, reddish-purple to

Light yellow (4A4-5) when fresh, and pale orange to light orange to brownish-orange very dark purple (old), occasionally fading to (5A3-4, 5C4, 6C6) after being dried, $\mathrm{KOH}(-)$. 


\begin{tabular}{|l|l|l|}
\hline & $\begin{array}{l}\text { H. lactifluorum } \\
\text { (Rogerson and Samuels 1994) }\end{array}$ & H. pseudolactifluorum \\
\hline Perithecia & $\begin{array}{l}\text { Ovate to obpyriform, deep orange to reddish- } \\
\text { purple, } 400-600 \times 200-450 \mu \mathrm{m}\end{array}$ & $\begin{array}{l}\text { Pyriform to subglobose, yellowish-brown to } \\
\text { dark brown (5E6, 6E6, 6F6-8), 262-484 } \\
136-284 \mu \mathrm{m}\end{array}$ \\
\hline $\begin{array}{l}\text { Embedded } \\
\text { type }\end{array}$ & Immersed except for papilla & $\begin{array}{l}\text { Semi-immersed to immersed except for } \\
\text { papilla }\end{array}$ \\
\hline Papilla & Averaging 120 $\mu \mathrm{m}$ high, $120 \mu \mathrm{m}$ wide & $\begin{array}{l}129-177 \mu \mathrm{m} \text { high and } 135-284 \mu \mathrm{m} \text { wide at } \\
\text { base }\end{array}$ \\
\hline Asci & Long cylindrical, 200-260 $\times 5-10 \mu \mathrm{m}$ & Cylindrical, 147-222 $\times 4-8.5 \mu \mathrm{m}$ \\
\hline Ascospores & Fusiform, 1-septate, 35-40 $\times 4.5-7 \mu \mathrm{m}$ & Fusiform, 1-septate, 30-38 $\times 5.5-8 \mu \mathrm{m}$ \\
\hline Apiculi & $4.5-7.5 \mu m$ long & $4-6 \mu \mathrm{m}$ long \\
\hline Hosts & Russula and Lactarius spp. & Russula sp. \\
\hline Distribution & North America & P.R. China (Yunnan) \\
\hline & & \\
\hline
\end{tabular}

\section{Etymology}

Referring to the most closely-related species, Hypomyces lactifluorum.

\section{Distribution}

PR CHINA (Yunnan).

\section{Host}

On the fruiting bodies of Russula sp. that grew on the humus layer in an evergreen broad-leaved forest of a rainforest. The host mushrooms: basidiocarps medium-sized and infundibuliform, pilei 63-77 mm in diameter. As serious degradation has occurred, the colour and other characters of the host mushrooms cannot be determined. Molecular phylogenetic evidence indicates it is a Russula species.

\section{Notes}

Only sexual morph had been discovered on the hosts (Russula sp.) of the new species.

\section{Analysis}

\section{Phylogenetic analyses}

\section{Parasitic fungus: Hypomyces pseudolactifluorum sp. nov}

The combined ITS+LSU+TEF1- $\alpha+$ RPB2 sequence dataset (excluding the outgroup taxa) contains 3,262 characters (709 for ITS, 893 for LSU, 921 for TEF1- $\alpha$ and 739 for RPB2) from 56 Hypomyces species and two Trichoderma species. Amongst them, 2,246 
characters are constant, 209 variable characters are parsimony-uninformative and 807 characters are parsimony-informative. The $\mathrm{ML}$ and $\mathrm{BI}$ analyses resulted in trees with similar topology and support values and the ML tree is shown in Fig. 2.

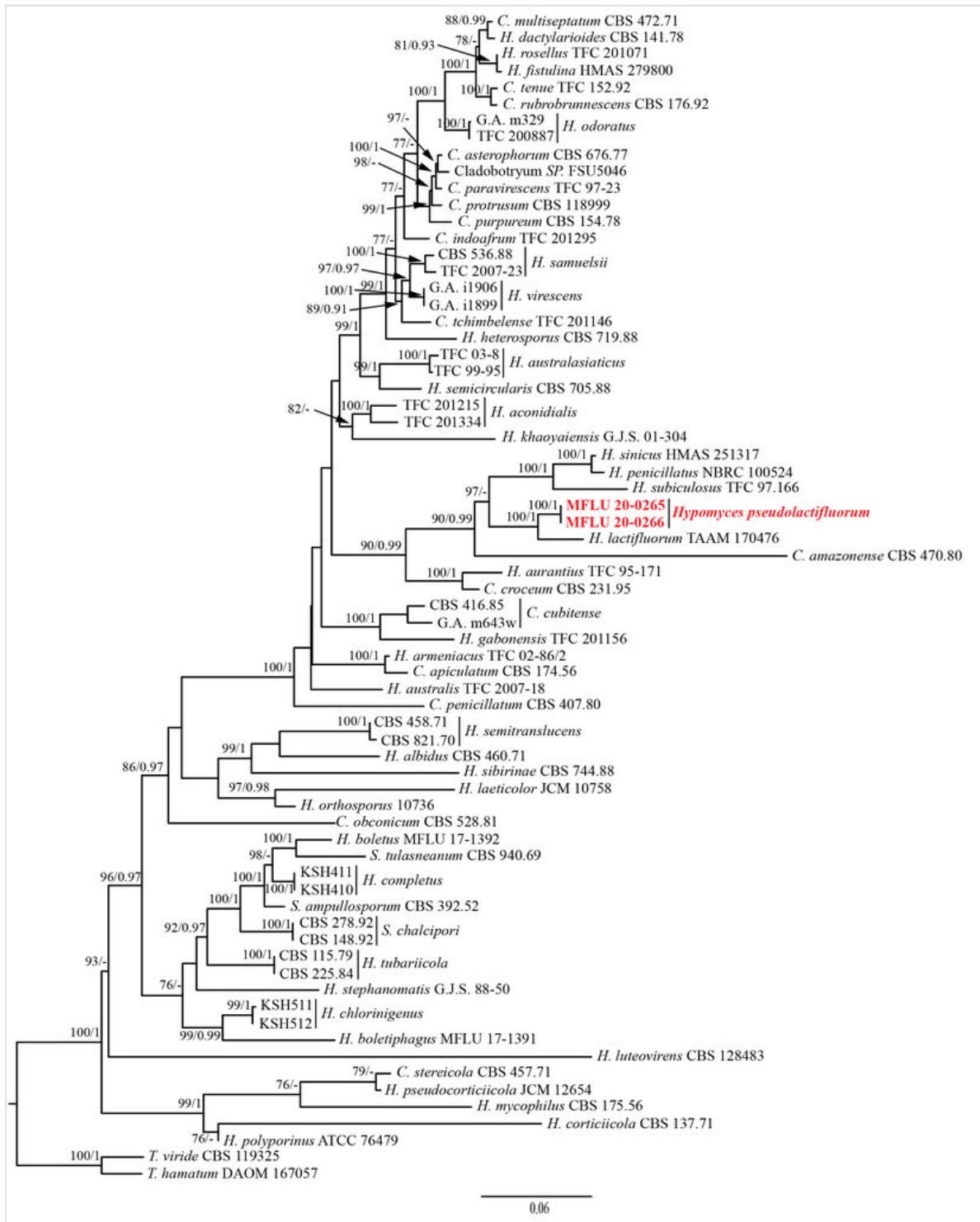

Figure 2. doi

ML tree of Hypomyces pseudolactifluorum sp. nov. and its allies generated from a combined ITS, LSU,TEF1- $\alpha$ and RPB2 gene sequence dataset. Supporting values of MLBP (left, greater than $75 \%$ ) and BIBP (right, greater than 0.9 ) are shown at the nodes, respectively. The new species is marked in red. 
In the phylogenetic tree, the parasitic fungi MFLU 20-0265 and MFLU 20-0266 are clustered together and formed a distinct lineage with the same branch length and strong supportive values $(\mathrm{MLBP}=100 \%$, BIPP $=1)$, which support them to be conspecific. The parasitic fungi are closely related $H$. lactifluorum and they form a sister clade also with strong supportive values (MLBP $=100 \%$, BIPP $=1)$. Comparing the gene sequences of the two species, there are 25 bp (4.3\%) differences across 582 bp in ITS, 28 bp (3.2\%) differences across 870 bp in LSU, 24 bp (2.6\%) differences across 921 bp in TEF1- $\alpha$ and $24 \mathrm{bp}(3.2 \%)$ differences across $739 \mathrm{bp}$ in RPB2 (Suppl. material 1). Following the recommendations from Jeewon and Hyde (2016), we assign the parasitic fungi as $H$. pseudolactifluorum sp. nov.

\section{The host mushroom: Russula sp.}

According to the ITS phylogenetic tree of the host mushroom and its allies, the host mushroom (MFLU 20-0265) is clustered together with Russula leucocarpa (HGAS-MF 009910 and HGAS-MF 009916) (MLBP = 100\%) in subsect. Lactarioideae. However, their ITS sequences have $24 \mathrm{bp}$ (3.5\%) differences across $694 \mathrm{bp}$, which indicated they may be two distinct species. Due to lack of sufficient morphological evidence, the host mushroom was temporarily identified as Russula sp. (Fig. 3).

\section{Discussion}

Zeng and Zhuang (2016) described $H$. amaniticola on Amanita sp. and $H$. completiopsis and $H$. yunnanensis on Boletus sp., also from China. Though with similar colour and shapes of perithecia, the host of $H$. pseudolactifluorum sp. nov. is decidedly different from those of these three species. Furthermore, $H$. pseudolactifluorum sp. nov. (KOH-) has smaller perithecia and larger ascospores than those of $\mathrm{H}$. completiopsis $\left(\mathrm{KOH}^{+}\right)$and $\mathrm{H}$. pseudolactifluorum sp. nov. has larger perithecia, asci and ascospores than those of $H$. amaniticola $\left(\mathrm{KOH}^{+}\right)$and $\mathrm{H}$. yunnanensis $(\mathrm{KOH}-)$. Unfortunately, these three species all lack molecular data.

With the rapid development of mushroom industries, fungal pathogens on mushrooms have received more and more attention (Hyde et al. 2019). The fungicolous fungi Hypomyces is an important group of mushroom pathogens. Many Hypomyces species, for example, $H$. aurantius, $H$. perniciosus, $H$. rosellus, $H$. odoratus etc., have all been recorded as the causes of Cobweb or Web bubble disease which seriously influence mushroom industries (Fletcher and Gaze 2007, Carrasco et al. 2017, Zhang et al. 2017, Zhang et al. 2017). Russula is the largest subgenus in agaric with approximately 800 species (Li et al. 2020) and many Russula species are important edible mushrooms. Since growing on Russula sp., H. pseudolactifluorum sp. nov., as well as H. lactifluorum from North America (Rogerson and Samuels 1994), could be one of the potential pathogens of some Russula species in Asia. 


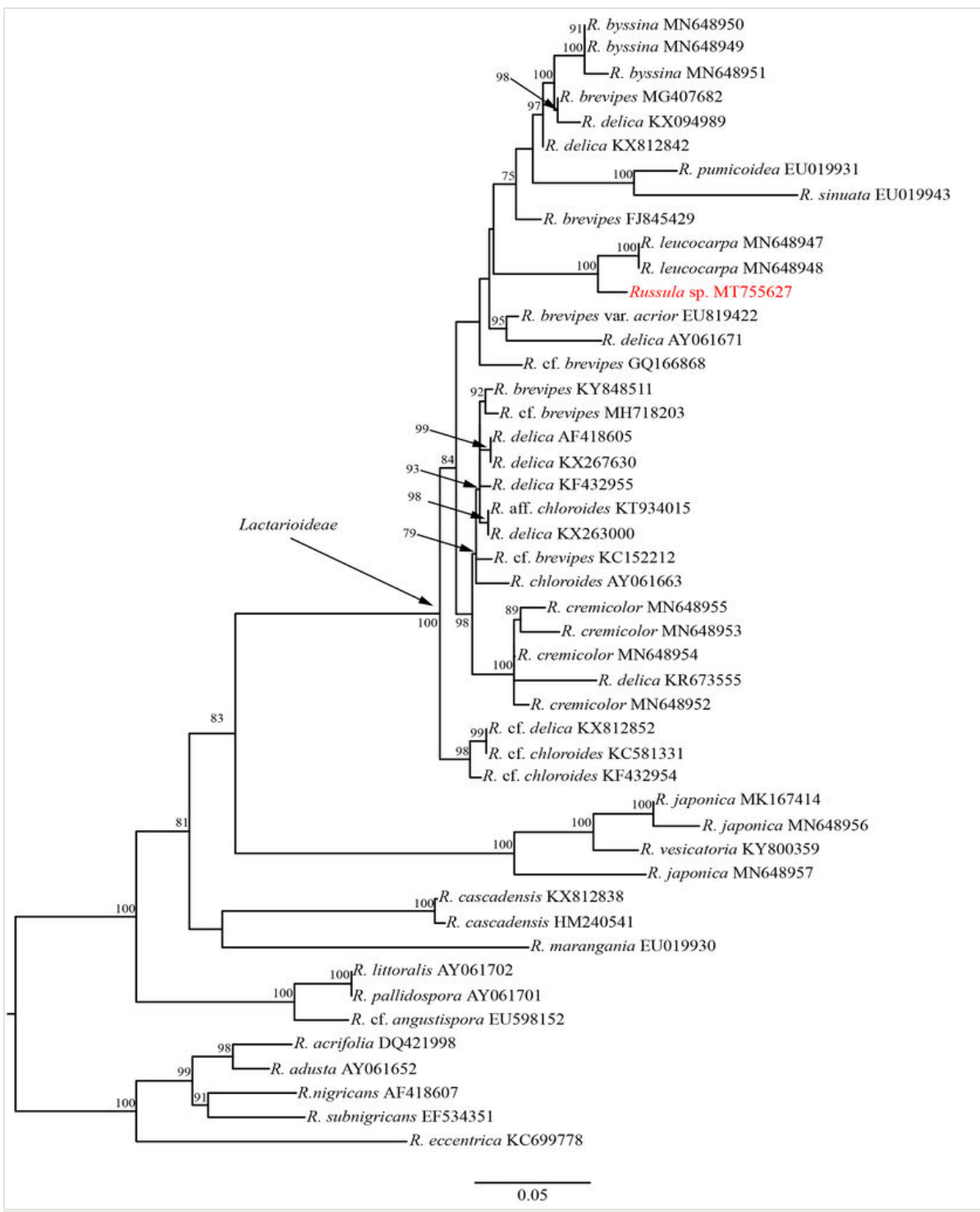

Figure 3. doi

$\mathrm{ML}$ tree of Russula sp. (in red) and it allies inferred from the ITS sequence dataset. Five species of Russula subg. Compactae were used as the outgroup taxa. Supporting values of MLBP (greater than $75 \%$ ) are shown at the nodes.

\section{Acknowledgements}

The research is supported by the Second Tibetan Plateau Scientific Expedition and Research (STEP) Program (Grant No. 2019QZKK0503); the Open Research Project of "Cross-Cooperative Team" of the Germplasm Bank of Wild Species, Kunming Institute of 
Botany, Chinese Academy of Sciences (Grant No. 292019312511043); Science and Technology Service Network Initiative of the Chinese Academy of Sciences (KFJ-STSQYZD-171); the Biodiversity Survey and Assessment Project of the Ministry of Ecology and Environment, PR China (2019HJ2096001006) and Impact of climate change on fungal diversity and biogeography in the Greater Mekong Subregion (Grant No. RDG6130001).

\section{References}

- $\quad$ Aghajani H, Hojjati SM, Tajick-Ghanbari MA, Puormajidian MR, Borhani A (2017) Molecular Identification of Ectomycorrhizal Fungal Communities Associated with Oriental Beech Trees (Fagus orientalis Lipsky) in Hyrcanian Forest of Iran. Iranian Journal of Science and Technology, Transactions A: Science 43 (1): 25-32.

https://doi.org/10.1007/s40995-017-0435-2

- Bazzicalupo AL (2018) Evaluating morphology and geographic range extent of genetically delimited species of mushrooms. The University of British Columbia, Vancouver, 1-247 pp.

- $\quad$ Buyck B, Duhem B, Das K, Jayawardena RS, Niveiro N, Pereira OL, Prasher IB, Adhikari S, Albertó EO, Bulgakov TS, Castañeda-Ruiz RF, Hembrom ME, Hyde KD, Lewis DP, Michlig A, Nuytinck J, Parihar A, Popoff OF, Ramirez NA, da Silva M, Verma RK, Hofstetter V (2017) Fungal biodiversity profiles 21-30. Cryptogamie Mycologie 38 (1): 101-146. https://doi.org/10.7872/crym/v38.iss1.2017.101

- $\quad$ Carbone I, Kohn L (1999) A method for designing primer sets for speciation studies in filamentous Ascomycetes . Mycologia 91: 553-556. https://doi.org/10.2307/3761358

- $\quad$ Carrasco J, Navarro M, Gea F (2017) Cobweb, a serious pathology in mushroom crops: A review. Spanish Journal of Agricultural Research 15 (2). https://doi.org/10.5424/sjar/ 2017152-10143

- $\quad$ Chen J, Fu X (1989) The verticillium-like genera parasitic on mushrooms. Acta Mycologica Sinica 8: 123-132. [In Chinese].

- Chen ZH, Zhang P (2019) Atlas of macrofungi in Hunan. Normal University Press, Changsha, 1-426 pp. [In Chinese].

- Chernomor O, von Haeseler A, Minh BQ (2016) Terrace Aware Data Structure for Phylogenomic Inference from Supermatrices. Systematic Biology 65 (6): 997-1008. https://doi.org/10.1093/sysbio/syw037

- $\quad$ Currie CR, Wong B, Stuart AE, Schultz TR, Rehner SA, Mueller UG, Sung G, Spatafora JW, Straus NA (2003) Ancient tripartite coevolution in the attine ant-microbe symbiosis. Science 299: 386-388. https://doi.org/10.1126/science.1078155

- $\quad$ Darriba D, Taboada GL, Doallo R, Posada D (2012) jModelTest 2: more models, new heuristics and parallel computing. Nature Methods 9 (8): 772-772.

https://doi.org/10.1038/nmeth.2109

- Doyle J (1987) A rapid DNA isolation procedure for small quantities of fresh leaf tissue. Phytochem Bull 19: 11-15.

- Eberhardt U (2002) Molecular kinship analyses of the agaricoid Russulaceae: Correspondence with mycorrhizal anatomy and sporocarp features in the genus Russula . Mycological Progress 1 (2): 201-223. https://doi.org/10.1007/ s11557-006-0019-6 
- $\quad$ Fletcher JT, Gaze RH (2007) Mushroom Pest and Disease Control: A Colour Handbook. Academic Press, San Diego https://doi.org/10.1201/b15139

- $\quad$ Fries E (1825) Systema Orbis Vegetabilis. Primas lineas novæ constructionis periclitatur EF Pars I. Plantæ Homonemeæ. Lundae : Typographia Academica, Lund, 369 pp.

- Hall T (1999) BioEdit: a user-friendly biological sequence alignment editor and analysis program for Windows 95/98/NT. Nucleic Acids Symposium Series 41: 95-98.

- Hoyos-Carvajal L, Orduz S, Bissett J (2009) Genetic and metabolic biodiversity of Trichoderma from Colombia and adjacent neotropic regions. Fungal Genetics and Biology 46: 615-631. https://doi.org/10.1016/j.fgb.2009.04.006

- Huelsenbeck J, Rannala B (2004) Frequentist Properties of Bayesian Posterior Probabilities of Phylogenetic Trees Under Simple and Complex Substitution Models. Systematic Biology 53 (6): 904-913. https://doi.org/10.1080/10635150490522629

- Huelsenbeck JP, Ronquist F (2001) MRBAYES: Bayesian inference of phylogenetic trees. Bioinformatics 17: 754-755. https://doi.org/10.1093/bioinformatics/17.8.754

- Hyde K, Xu J, Rapior S, Jeewon R, Lumyong S, Niego A, Abeywickrama P, Aluthmuhandiram JS, Brahamanage R, Brooks S, Chaiyasen A, Chethana KWT, Chomnunti P, Chepkirui C, Chuankid B, de Silva N, Doilom M, Faulds C, Gentekaki E, Gopalan V, Kakumyan P, Harishchandra D, Hemachandran H, Hongsanan S, Karunarathna A, Karunarathna S, Khan S, Kumla J, Jayawardena R, Liu J, Liu N, Luangharn T, Macabeo A, Marasinghe D, Meeks D, Mortimer P, Mueller P, Nadir S, Nataraja K, Nontachaiyapoom S, O'Brien M, Penkhrue W, Phukhamsakda C, Ramanan US, Rathnayaka A, Sadaba R, Sandargo B, Samarakoon B, Tennakoon D, Siva R, Sriprom W, Suryanarayanan TS, Sujarit K, Suwannarach N, Suwunwong T, Thongbai B, Thongklang N, Wei D, Wijesinghe SN, Winiski J, Yan J, Yasanthika E, Stadler M (2019) The amazing potential of fungi: 50 ways we can exploit fungi industrially. Fungal Diversity 97: 1-136. https://doi.org/10.1007/s13225-019-00430-9

- Hyde K, Norphanphoun C, Maharachchikumbura S, Bhat D, Jones E, Bundhun D, Chen Y, Bao D, Boonmee S, Calabon M, Chaiwan N, Chethana K, Dai D, Dayarathne M, Devadatha B, Dissanayake A, Dissanayake L, Doilom M, Dong W, Fan X, Goonasekara I, Hongsanan S, Huang S, Jayawardena R, Jeewon R, Karunarathna A, Konta S, Kumar V, Lin C, Liu J, Liu N, Luangsa-ard J, Lumyong S, Luo Z, Marasinghe D, McKenzie E, Niego A, Niranjan M, Perera R, Phukhamsakda C, Rathnayaka A, Samarakoon M, Samarakoon S, Sarma V, Senanayake I, Shang Q, Stadler M, Tibpromma S, Wanasinghe D, Wei D, Wijayawardene N, Xiao Y, Yang J, Zeng X, Zhang S, Xiang M (2020) Refined families of Sordariomycetes . Mycosphere 11: 305-1059. https://doi.org/10.5943/mycosphere/11/1/7

- Jaklitsch W, Samuels G, Dodd S, Lu B, Druzhinina I (2006) Hypocrea rufa/Trichoderma viride: a reassessment, and description of five closely related species with and without warted conidia. Studies in Mycology 56: 135-177. https://doi.org/10.3114/sim.

2006.56.04

- Jeewon R, Hyde KD (2016) Establishing species boundaries and new taxa among fungi: recommendations to resolve taxonomic ambiguities. Mycosphere 7 (11): 1669-1677. https://doi.org/10.5943/mycosphere/7/11/4

- $\quad$ Katoh K, Kuma K, Toh H, Miyata T (2005) MAFFT version 5: improvement in accuracy of multiple sequence alignment. Nucleic acids research 33: 511-8.

https://doi.org/10.1093/nar/gki198 
- $\quad$ Kim CS, Jo JW, Kwag Y, Sung G, Lee S, Kim S, Shin C, Han S (2015) Mushroom Flora of Ulleung-gun and a Newly Recorded Bovista Species in the Republic of Korea.

Mycobiology 43 (3): 239-257. https://doi.org/10.5941/myco.2015.43.3.239

- Kornerup A, Wanscher JH (1978) Methuen Handbook of Colour, 3rd edn. London: Eyre Methuen

- Kranabetter JM, Friesen J, Gamiet S, Kroeger P (2009) Epigeous fruiting bodies of ectomycorrhizal fungi as indicators of soil fertility and associated nitrogen status of boreal forests. Mycorrhiza 19 (8): 535-548. https://doi.org/10.1007/s00572-009-0255-0

- Lebel T, Tonkin J (2007) Australasian species of Macowanites are sequestrate species of Russula (Russulaceae, Basidiomycota). Australian Systematic Botany 20 (4): 355-381. https://doi.org/10.1071/sb07007

- $\quad$ Lechat C, Gardiennet A, Fournier J (2017) First report of a lichenicolous species of Hypomyces (Hypocreaceae), H. peltigericola sp. nov. Ascomycete. org 9: 23-26.

- $\quad$ Li GJ, Deng CY, Shi LY, Wang J, Meng QF, Li SM (2020) Three new species of Russula subsect. Lactarioideae from China. Mycosystema 39 (4): 618-636.

- $\quad$ Liu XL, Bau T, Wang XH (2017) Species diversity of Russula from the Greater and Lesser Hinggan Mountains in Northeast China. Mycosystema 36 (10): 1355-1368.

- Liu YJ, Whelen S, Hall BD (1999) Phylogenetic relationships among ascomycetes: evidence from an RNA polymerse II subunit. Molecular Biology and Evolution 16: 1799-1808. https://doi.org/10.1093/oxfordjournals.molbev.a026092

- $\quad$ Looney B, Ryberg M, Hampe F, Sánchez-García M, Matheny PB (2016) Into and out of the tropics: global diversification patterns in a hyperdiverse clade of ectomycorrhizal fungi. Molecular Ecology 25 (2): 630-647. https://doi.org/10.1111/mec.13506

- Looney B, Meidl P, Piatek M, Miettinen O, Martin F, Matheny PB, Labbé J (2018) Russulaceae: a new genomic dataset to study ecosystem function and evolutionary diversification of ectomycorrhizal fungi with their tree associates. New Phytologist 218 (1): 54-65. https://doi.org/10.1111/nph.15001

- Luo J, Zhuang W (2012) Re-examinations of the Hypomyces specimens on deposit in HMAS. Mycosystema 31: 784-788.

- Ma J (2008) Study on morphology and taxonomy of mycoparasite fungi. Jilin Agricultural University, Changchun, 73 pp. [In Chinese].

- $\quad$ McKnight KH, McKnight VB (1997) A field guide to mushrooms: North America. Vol. 34. Houghton Mifflin Harcourt.

- $\quad$ Miller S, Buyck B (2002) Molecular phylogeny of the genus Russula in Europe with a comparison of modern infrageneric classifications. Mycological Research 106 (3): 259-276. https://doi.org/10.1017/s0953756202005610

- $\quad$ Nguyen L, Schmidt H, von Haeseler A, Minh BQ (2014) IQ-TREE: A Fast and Effective Stochastic Algorithm for Estimating Maximum-Likelihood Phylogenies. Molecular Biology and Evolution 32 (1): 268-274. https://doi.org/10.1093/molbev/msu300

- $\quad$ Otto A, Laub A, Wendt L, Porzel A, Schmidt J, Palfner G, Becerra J, Krüger D, Stadler M, Wessjohann L, Westermann B, Arnold N (2016) Chilenopeptins A and B, peptaibols from the Chilean Sepedonium aff. chalcipori KSH 883. Journal of Natural Products 79: 929-938. https://doi.org/10.1021/acs.jnatprod.5b01018

- $\quad$ Palmer J, Lindner D, Volk T (2008) Ectomycorrhizal characterization of an American chestnut (Castanea dentata)-dominated community in Western Wisconsin. Mycorrhiza 19 (1): 27-36. https://doi.org/10.1007/s00572-008-0200-7 
- $\quad$ Park MS, Lee H, Oh S, Jung PE, Seok SJ, Fong J, Lim YW (2014) Species delimitation of three species within the Russula subgenus Compacta in Korea: $R$. eccentrica, $R$. nigricans, and $R$. subnigricans. Journal of Microbiology 52 (8): 631-638.

https://doi.org/10.1007/s12275-014-4168-z

- Põldmaa K (1996) A new species of Hypomyces and three of Cladobotryum from Estonia, with a discussion of their taxonomic position. Mycotaxon 59: 389-405.

- Põldmaa K, Larsson E, Kõljalg U (2000) Phylogenetic relationships in Hypomyces and allied genera, with emphasis on species growing on wood-decaying homobasidiomycetes. Canadian Journal of Botany 77 (12): 1756-1768. https://doi.org/10.1139/b99-148

- $\quad$ Põldmaa K (2011) Tropical species of Cladobotryum and Hypomyces producing red pigments. Studies in Mycology 68: 1-34. https://doi.org/10.3114/sim.2011.68.01

- Rehner S, Samuels G (1994) Taxonomy and phylogeny of Gliocladium analysed from nuclear large subunit ribosomal DNA sequences. Mycological Research 98 (6): 625-634. https://doi.org/10.1016/s0953-7562(09)80409-7

- $\quad$ Rehner SA, Buckley E (2005) A Beauveria phylogeny inferred from nuclear ITS and EF1- sequences: evidence for cryptic diversification and links to Cordyceps teleomorphs. Mycologia 97 (1): 84-98. https://doi.org/10.3852/mycologia.97.1.84

- $\quad$ Rogerson C, Samuels G (1985) Species of Hypomyces and Nectria occurring on Discomycetes. Mycologia 77 (5). https://doi.org/10.2307/3793285

- Rogerson C, Samuels G (1989) Boleticolous species of Hypomyces. Mycologia 81 (3). https://doi.org/10.2307/3760079

- Rogerson C, Samuels G (1993) Polyporicolous species of Hypomyces. Mycologia 85 (2). https://doi.org/10.2307/3760461

- Rogerson C, Samuels G (1994) Agaricicolous species of Hypomyces. Mycologia 86 (6). https://doi.org/10.2307/3760597

- Ronquist F, Teslenko M, van der Mark P, Ayres D, Darling A, Höhna S, Larget B, Liu L, Suchard M, Huelsenbeck J (2012) MrBayes 3.2: Efficient Bayesian phylogenetic inference and model choice across a large model space. Systematic Biology 61 (3): 539-542. https://doi.org/10.1093/sysbio/sys029

- Rossman A, Rogersson C (1981) A new species of Hypomyces (Hypocreaceae) with phragmosporous ascospores. Brittonia 33 (3). https://doi.org/10.2307/2806428

- Rossman A, Samuels G, Rogerson C, Lowen R (1999) Genera of Bionectriaceae, Hypocreaceae and Nectriaceae (Hypocreales, Ascomycetes). Studies in Mycology 42: $1-260$.

- Rossman A, Seifert K, Samuels G, Minnis A, Schroers H, Lombard L, Crous P, Põldmaa K, Cannon P, Summerbell R, Geiser D, Zhuang W, Hirooka Y, Herrera C, SalgadoSalazar C, Chaverri P (2013) Genera in Bionectriaceae, Hypocreaceae, and Nectriaceae (Hypocreales) proposed for acceptance or rejection. IMA Fungus 4 (1): 41-51. https://doi.org/10.5598/imafungus.2013.04.01.05

- Samuels G, Doi Y, Rogerson C (1990) Hypocreales. Memoirs of the New York Botanical Garden 59: 6-108.

- $\quad$ Sun J, Liu X, McKenzie EC, Jeewon R, Liu J(, Zhang X, Zhao Q, Hyde K (2019a) Fungicolous fungi: terminology, diversity, distribution, evolution, and species checklist. Fungal Diversity 95 (1): 337-430. https://doi.org/10.1007/s13225-019-00422-9 
- $\quad$ Sun J, Liu X, Jeewon R, Lin C, Tian Q, Zhao Q, Xiao X, Hyde K, Nilthong S (2019b) Fifteen fungicolous Ascomycetes on edible and medicinal mushrooms in China and Thailand. Asian Journal of Mycology 2 (1): 129-169. https://doi.org/10.5943/ajom/2/1/7

- $\quad$ Tamm H, Põldmaa K (2013) Diversity, host associations, and phylogeography of temperate aurofusarin-producing Hypomyces / Cladobotryum including causal agents of cobweb disease of cultivated mushrooms. Fungal Biology 117 (5): 348-367.

https://doi.org/10.1016/j.funbio.2013.03.005

- $\quad$ Teng S (1934) Notes on Hypocreales from China. Sinensia 4: 269-298.

- $\quad$ Teng S (1963) Fungi of China. 1st Edition. Science Press, Beijing, 808 pp. [In Chinese].

- Tulasne L, Tulasne C (1860) De quelques sphéries fongicoles, à propos d'un mémoire de M. Antoine de Bary sur les Nyctalis . Annales des Sciences Naturelles Botanique 13: 5-19.

- Vilgalys R, Hester M (1990) Rapid genetic identification and mapping of enzymatically amplified ribosomal DNA from several Cryptococcus species. Journal of Bacteriology 172 (8): 4238-4246. https://doi.org/10.1128/jb.172.8.4238-4246.1990

- Vu D, Groenewald M, de Vries M, Gehrmann T, Stielow B, Eberhardt U, Al-Hatmi A, Groenewald JZ, Cardinali G, Houbraken J, Boekhout T, Crous PW, Robert V, Verkley GJ (2019) Large-scale generation and analysis of filamentous fungal DNA barcodes boosts coverage for kingdom fungi and reveals thresholds for fungal species and higher taxon delimitation. Studies in Mycology 92: 135-154. https://doi.org/10.1016/j.simyco. 2018.05.001

- Wei I, Kirschner R (2017) Two fungicolous anamorphic species of Hypomyces s. lat. from Taiwan. Fungal Science 32: 15-25.

- White TJ, Bruns T, Lee S, Taylor J (1990) Amplification and direct sequencing of fungal ribosomal RNA genes for phylogenetics. PCR Protocols 315-322.

https://doi.org/10.1016/b978-0-12-372180-8.50042-1

- Wijayawardene N, Hyde K, Tibpromma S, Wanasinghe D, Thambugala K, Tian Q, Wang $Y(2017)$ Towards incorporating asexual fungi in a natural classification: checklist and notes 2012-2016. Mycosphere 8: 1457-1555.

https://doi.org/10.5943/mycosphere/8/9/10

- Wisitrassameewong K, NUYTINCK J, HYDE KD, VERBEKEN A (2014) Lactarius subgenus Russularia (Russulaceae) in Southeast Asia: 1. Species with very distant gills. Phytotaxa 158 (1): 23-42. https://doi.org/10.11646/phytotaxa.158.1.2

- $\quad$ Yin JH, Zhang P, Gong QF, Chen ZH (2008) Sequence analysis of the internal transcribed spacer of gene coding for rDNA in Russula subnigricans and $R$. nigricans . Mycosystema 27 (2): 237-242. [In In Chinese].

- Zare R, Gams W (2016) More white verticillium-like anamorphs with erect conidiophores. Mycological Progress 15: 993-1030. https://doi.org/10.1007/ s11557-016-1214-8

- $\quad$ Zeng Z, Zhuang W (2015) Current understanding of the genus Hypomyces (Hypocreales). Mycosystema 34: 809-816. [In Chinese].

- $\quad$ Zeng Z, Zhuang W (2016) Three new species and two new Chinese records of Hypomyces (Hypocreales). Mycosystema 35: 1048-1055. [In Chinese].

- $\quad$ Zeng Z, Wing X, Zhuang W (2017) Three new Chinese records of Hypomyces (Hypocreales). Mycosystema 36: 522-527. 
- $\quad$ Zeng ZQ, Zhuang WY (2019) Two new species and a new Chinese record of Hypocreaceae as evidenced by morphological and molecular data. Mycobiology 47 (3): 280-291. https://doi.org/10.1080/12298093.2019.1641062

- $\quad$ Zhang C, Kakishima M, Xu J, Wang Q, Li Y (2017) The effect of Hypomyces perniciosus on the mycelia and basidiomes of Agaricus bisporus . Microbiology 163 (9): 1273-1282. https://doi.org/10.1099/mic.0.000521

- $\quad$ Zhang CL, Xu JZ, Kakishima M, Li Y (2017) First Report of Wet Bubble Disease Caused by Hypomyces perniciosus on Pleurotus citrinopileatus in China. Plant Disease 101 (7): 1321-1321. https://doi.org/10.1094/pdis-02-17-0179-pdn

- $\quad$ Zhuang W, Chen S, Zeng Z, Zheng H (2012) A new species of Hypomyces (Hypocreales) on Schizophyllum sp. from China. Mycosystema 31: 821-826.

\section{Supplementary material}

Suppl. material 1: Hypomyces pseudolactifluorum sp. nov. (Hypocreales: Hypocreaceae) on Russula sp. from Yunnan, PR China doi

Authors: FENG-MING YU, RUVISHIKA S. JAYAWARDENA, JIAN-WEI LIU, KEVIN D. HYDE, QI ZHAO

Data type: word

Brief description: Sequence differences of ITS, LSU, TEF1- $\alpha$ and RPB2 genes between $H$. lactifluorum (TAAM 170476) and $H$. pseudolactifluorum sp. nov.. The locus' numbers refer to the nucleotide positions of the gene sequences of $H$. lactifluorum from GenBank. Gap is replaced by $\because-$

Download file $(20.36 \mathrm{~kb})$ 